\title{
The Modified Trial Equation Method for Fractional Wave Equation and Time Fractional Generalized Burgers Equation
}

\author{
Hasan Bulut, ${ }^{1}$ Haci Mehmet Baskonus, ${ }^{2}$ and Yusuf Pandir ${ }^{3}$ \\ ${ }^{1}$ Department of Mathematics, Faculty of Science, Firat University, 23100 Elazig, Turkey \\ ${ }^{2}$ Department of Computer Engineering, Faculty of Engineering, University of Tunceli, 62100 Tunceli, Turkey \\ ${ }^{3}$ Department of Mathematics, Faculty of Science, Bozok University, 66100 Yozgat, Turkey \\ Correspondence should be addressed to Haci Mehmet Baskonus; hmbaskonus@gmail.com
}

Received 24 May 2013; Revised 15 July 2013; Accepted 15 July 2013

Academic Editor: Juan J. Trujillo

Copyright (C) 2013 Hasan Bulut et al. This is an open access article distributed under the Creative Commons Attribution License, which permits unrestricted use, distribution, and reproduction in any medium, provided the original work is properly cited.

\begin{abstract}
The fractional partial differential equations stand for natural phenomena all over the world from science to engineering. When it comes to obtaining the solutions of these equations, there are many various techniques in the literature. Some of these give to us approximate solutions; others give to us analytical solutions. In this paper, we applied the modified trial equation method (MTEM) to the one-dimensional nonlinear fractional wave equation (FWE) and time fractional generalized Burgers equation. Then, we submitted 3D graphics for different value of $\alpha$.
\end{abstract}

\section{Introduction}

All over the world, a physical event may depend not only on the time but also on the previous process, which can be successfully formed by using the theory of derivatives and integrals of fractional order. These processes represent different physical problems in the manner of variable order. In this sense, the fractional differential equations have been used for the definition of nonlinear phenomena in applied science, physics, chemistry, engineering, and other areas of science. In order to solve these problems, a general method cannot be defined even in the most useful works. Also, a remarkable progress has been become in the construction of the approximate solutions for fractional nonlinear partial differential equations [1-3]. Several powerful methods [413] have been proposed to obtain approximate and exact solutions of fractional differential equations, such as the Sumudu transform method, the Homotopy analysis method, and the homotopy perturbation method.

Liu introduced a new approach called the complete discrimination system for a polynomial to classify the traveling wave solutions as nonlinear evolution equations and applied this idea to some nonlinear partial differential equations $[14,15]$. So, to the best of our knowledge, the modified trial equation method has not been widely applied for studying the invariance properties of fractional PDEs. Furthermore, some authors $[16,17]$ used the trial equation method proposed by Liu. However, we established a new modified trial equation method to obtain 1-soliton, singular soliton, hyperbolic function solutions $[18,19]$, elliptic integral function and Jacobi elliptic function solutions, or the others to nonlinear partial differential equations with generalized evolution in [20-22].

In Section 2, primarily we give some definitions and properties of the fractional calculus and also produce a new modified trial equation method for fractional nonlinear evolution equations with higher order nonlinearity. The power of this steerable method showed that this method can be applied to different equations. In Section 3, as an application, we solve the nonlinear fractional partial differential equation such as one-dimensional nonlinear fractional wave equation [23]:

$$
\frac{\partial^{\alpha} u}{\partial t^{\alpha}}+a u_{x x}+\beta u+\gamma u^{3}=0
$$

where $a, \beta$, and $\gamma$ are arbitrary constants and $\alpha$ is a parameter describing the order of the fractional time derivative. 
We consider time fractional generalized Burgers equation [27] described as follows:

$$
\frac{\partial^{\alpha} u}{\partial t^{\alpha}}-u_{x x}-\beta u^{p} u_{x}=0,
$$

where $0<\alpha \leq 1, p>0$ which occur in different areas in mathematical physics; here the time fractional derivative leads to subdiffusion and subdispersion, respectively, and extend the Lie symmetry analysis to derive their infinitesimals [24]. In this research, we obtain the classification of the wave solutions to (1) and (2) and derive some new solutions. Using the modified trial equation method, we found some new exact solutions of the fractional nonlinear physical problem. The purpose of this paper is to obtain exact solutions of the one-dimensional nonlinear fractional wave equation by modified trial equation method.

\section{Preliminaries}

In this part of the paper, it would be helpful to give some definitions and properties of the fractional calculus theory. Here, we shortly review the modified Riemann-Liouville derivative from the recent fractional calculus proposed by Jumarie $[25,26]$. Let $f:[0,1] \rightarrow \Re$ be a continuous function and $\alpha \in(0,1)$. The Jumarie modified fractional derivative of order $\alpha$ and $f$ may be defined by the expression of the following [23]:

$$
\begin{aligned}
& D_{x}^{\alpha} f(x) \\
& =\left\{\begin{array}{lr}
\frac{1}{\Gamma(-\alpha)} \int_{0}^{x}(x-\xi)^{-\alpha-1}[f(\xi)-f(0)] d \xi, & \alpha<0, \\
\frac{1}{\Gamma(1-\alpha)} \frac{d}{d x} & 0<\alpha<1, \\
\times \int_{0}^{x}(x-\xi)^{-\alpha}[f(\xi)-f(0)] d \xi, & \\
\left(f^{(n)}(\xi)\right)^{\alpha-n}, & n \leq \alpha \leq n+1, n \geq 1 .
\end{array}\right.
\end{aligned}
$$

In addition to this expression, we may give a summary of the fractional modified Riemann-Liouville derivative properties which are used further in this paper. Some of the useful formulas are given as [23]

$$
\begin{gathered}
D_{x}^{\alpha} k=0, \\
D_{x}^{\alpha} x^{\mu}= \begin{cases}0, & \mu \leq \alpha-1, \\
\frac{\Gamma(\mu+1)}{\Gamma(\mu-\alpha+1)} x^{\mu-x}, & \mu>\alpha-1 .\end{cases}
\end{gathered}
$$

In this paper, a new approach to the trial equation method will be given. In order to apply this method to fractional nonlinear partial differential equations, we consider the following steps.

Step 1. We consider time fractional partial differential equation in two variables and a dependent variable $u$ :

$$
P\left(u, D_{t}^{\alpha} u, u_{x}, u_{x x}, u_{x x x}, \ldots\right)=0,
$$

and take the wave transformation

$$
u(x, t)=u(\eta), \quad \eta=k x-\frac{\lambda t^{\alpha}}{\Gamma(1+\alpha)},
$$

where $\lambda \neq 0$. Substituting (6) into (5) yields a nonlinear ordinary differential equation:

$$
N\left(u, u^{\prime}, u^{\prime \prime}, u^{\prime \prime \prime}, \ldots\right)=0
$$

Step 2. Take trial equation as follows:

$$
\begin{gathered}
u^{\prime}=\frac{F(u)}{G(u)}=\frac{\sum_{i=0}^{n} a_{i} u^{i}}{\sum_{j=0}^{l} b_{j} u^{j}}=\frac{a_{0}+a_{1} u+a_{2} u^{2}+\cdots+a_{n} u^{n}}{b_{0}+b_{1} u+b_{2} u^{2}+\cdots+b_{l} u^{l}}, \\
u^{\prime \prime}=\frac{F(u)\left(F^{\prime}(u) G(u)-F(u) G^{\prime}(u)\right)}{G^{3}(u)},
\end{gathered}
$$

where $F(u)$ and $G(u)$ are polynomials. Substituting above relations into (7) yields an equation of polynomial $\Omega(u)$ of $u$ :

$$
\Omega(u)=\rho_{s} u^{s}+\cdots+\rho_{1} u+\rho_{0}=0 .
$$

According to the balance principle, we can get a relation of $n$ and $l$. We can compute some values of $n$ and $l$.

Step 3. Letting the coefficients of $\Omega(u)$ all be zero will yield an algebraic equations system:

$$
\rho_{i}=0, \quad i=0, \ldots, s .
$$

By solving this system, we will specify the values of $a_{0}, \ldots, a_{n}$ and $b_{0}, \ldots, b_{l}$.

Step 4. Reduce (8) to the elementary integral form

$$
\pm\left(\mu-\mu_{0}\right)=\int \frac{G(u)}{F(u)} d u .
$$

Using a complete discrimination system for polynomial to classify the roots of $F(u)$, we solve (12) with the help of Mathematica7 and classify the exact solutions to (7). In addition, we can write the exact traveling wave solutions to (5), respectively. For a better interpretation of results obtained in this way, we plotted 3D surfaces of (27), (40), (52), and (60) in Figures 1, 2, 3, and 4 by taking into consideration suitable parameter.

\section{Applications}

In this section, we applied the method to the one-dimensional nonlinear fractional wave equation and time fractional generalized Burgers equation.

Example 1. Firstly, we consider one-dimensional nonlinear fractional wave equation [23]. In the case of $\alpha=1$, (1) reduces to the classical nonlinear one-dimensional nonlinear wave equation. Many researchers have tried to get the exact solutions of this equation by using a variety of methods. 


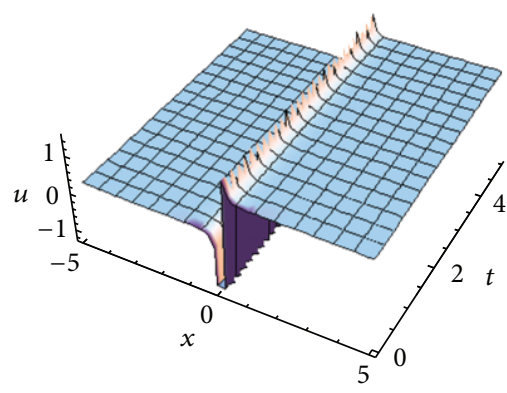

(a)

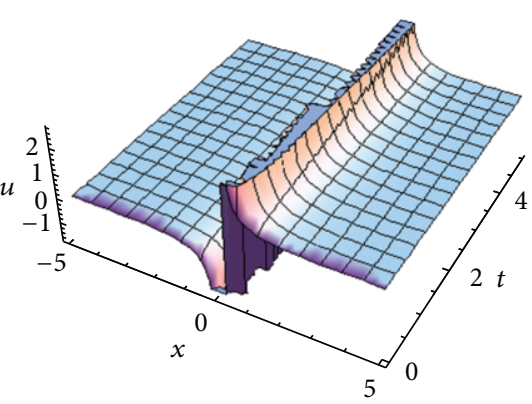

(b)

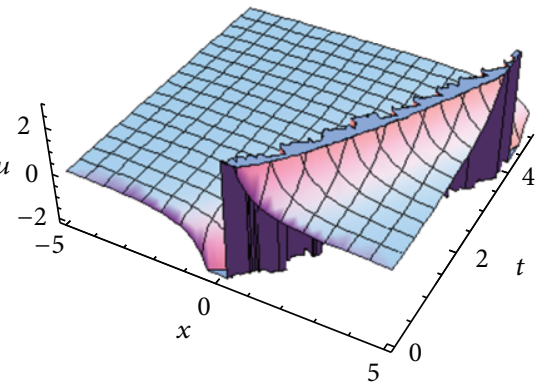

(c)

FIGURE 1: Graphics of the solution equation (27) corresponding to the values $\alpha=\beta=0.01, \alpha=\beta=0.25$, and $\alpha=\beta=0.75$ from left to right when $k=a_{1}=1, a_{2}=0.1, \gamma=-1,-5<x<5$, and $0<t<5$.

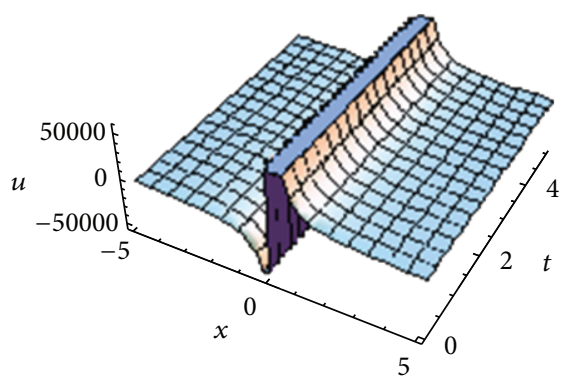

(a)

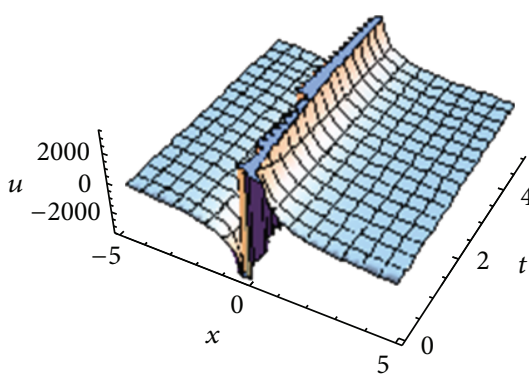

(b)

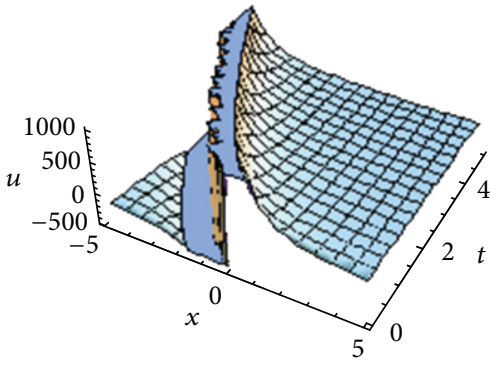

(c)

Figure 2: Graphics of the solution equation (40) corresponding to the values $\alpha=\beta=0.01, \alpha=\beta=0.25$, and $\alpha=\beta=0.75$ from left to right when $k=a_{1}=b_{0}=b_{1}=\gamma=0.01,-5<x<5$, and $0<t<5$.

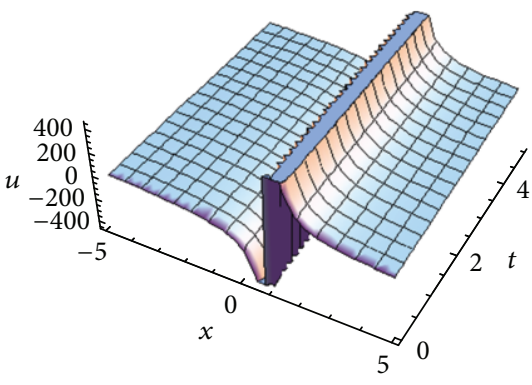

(a)

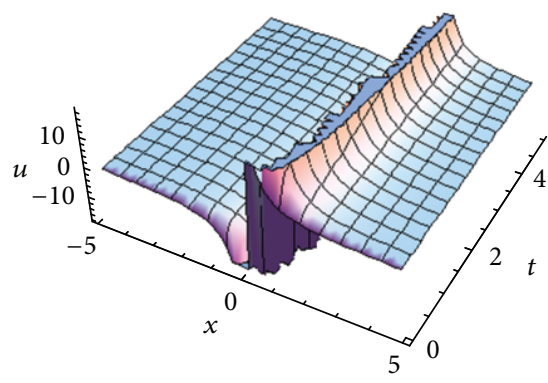

(b)

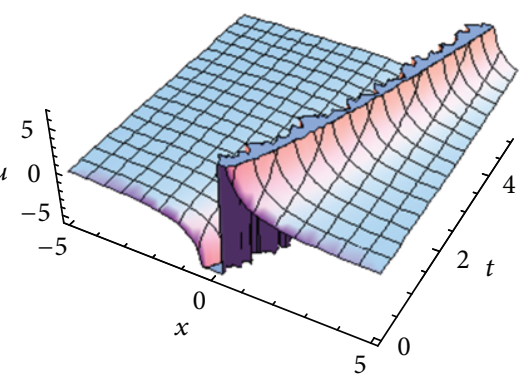

(c)

Figure 3: Graphics of the solution equation (52) corresponding to the values $\alpha=\beta=0.01, \alpha=\beta=0.25$, and $\alpha=\beta=0.75$ from left to right when $k=a_{2}=b_{0}=0.1,-5<x<5$, and $0<t<5$.

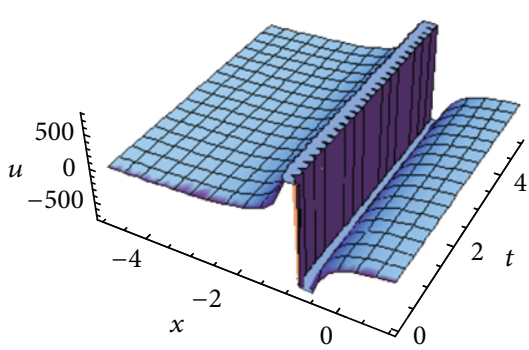

(a)

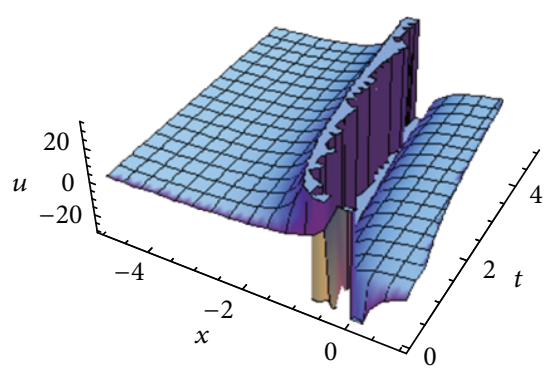

(b)

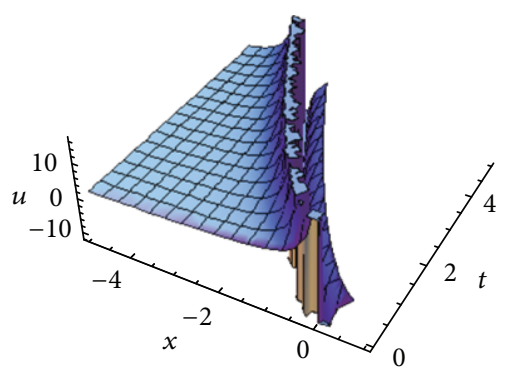

(c)

FIGURE 4: Graphics of the solution equation (60) corresponding to the values $\alpha=\beta=0.01, \alpha=\beta=0.25$, and $\alpha=\beta=0.75$ from left to right when $a_{1}=b_{0}=b_{1}=p=k=1,-5<x<1$, and $0<t<5$. 
Let us consider the travelling wave solutions of (1), and then and we perform the transformation $u(x, t)=u(\eta)$ and $\eta=k x-\lambda t^{\alpha} / \Gamma(1+\alpha)$ where $k$ and $\lambda$ are constants. Then, integrating this equation with respect to $\eta$ and setting the integration constant to zero. When it comes to converting fractional order differential equation into differential equation with integer order, we can perform the following:

$$
\begin{gathered}
\frac{d^{\alpha} u}{d t^{\alpha}}=\frac{d^{\alpha} u}{d \eta^{\alpha}} \frac{d^{\alpha} \eta}{d t^{\alpha}}=-\lambda u^{\prime}, \\
\frac{d^{2} u}{d x^{2}}=\frac{d}{d \eta}\left(\frac{d u}{d \eta}\right) \frac{d \eta}{d x}+\frac{d u}{d \eta} \frac{d}{d \eta}\left(\frac{d \eta}{d x}\right)=k^{3} u^{\prime \prime},
\end{gathered}
$$

so, when we use $d^{\alpha} u / d t^{\alpha}$ and $d^{2} u / d x^{2}$ in (1), we get ordinary differential equation as follows:

$$
-\lambda u^{\prime}+a k^{3} u^{\prime \prime}+\beta u+\gamma u^{3}=0 .
$$

When we rearrange to (8) and (9) for balance principle, we obtain the following:

$$
\begin{gathered}
u^{\prime}=\frac{a_{n}}{b_{l}} u^{n-l}+\cdots, \\
u^{\prime \prime}=\frac{\left(a_{n} n a_{n} b_{l}-b_{l} l a_{n}^{2}\right)}{b_{l}^{3}} u^{2 n-2 l-1}+\cdots .
\end{gathered}
$$

Balancing the highest order nonlinear terms of $u^{\prime \prime}$ and $u^{3}$ in (14), we get balance term for suitability

$$
2 n-2 l-1=3 \Longrightarrow n=l+2 \text {. }
$$

This resolution procedure is applied, and we obtain results as follows.

Case 1. If we take $l=0$ and $n=2$, then

$$
u^{\prime}=\frac{F(u)}{G(u)}=\frac{\sum_{i=0}^{n} a_{i} u^{i}}{\sum_{j=0}^{l} b_{j} u^{j}}=\frac{a_{0}+a_{1} u+a_{2} u^{2}}{b_{0}},
$$

and then

$$
\begin{aligned}
u^{\prime \prime} & =\frac{F(u)\left[F^{\prime}(u) G(u)-F(u) G^{\prime}(u)\right]}{G^{3}(u)}, \\
& =\frac{\left(a_{1}+2 a_{2} u\right)\left(a_{0}+a_{1} u+a_{2} u^{2}\right)}{b_{0}^{2}},
\end{aligned}
$$

where $a_{2} \neq 0$ and $b_{0} \neq 0$ When we use $u^{\prime}$ and $u^{\prime \prime}$ in (14), we get a system of algebraic equations for (14). Thus, we have a system of algebraic equations from the coefficients of the polynomial of $u$. Solving the algebraic equation system (14) by using Mathematica programming yields the following coefficients:

$$
\begin{gathered}
a_{0}=0, \quad a_{1}=\frac{\sqrt{\beta} b_{0}}{k \sqrt{2 a}}, \quad a_{2}= \pm \frac{\sqrt{-\gamma} b_{0}}{k \sqrt{2 a}}, \\
b_{0}=b_{0}, \quad \lambda=\frac{3 k \sqrt{\beta a}}{\sqrt{2}} .
\end{gathered}
$$

By substituting these coefficients into (12), we have

$$
\pm\left(\mu-\mu_{0}\right)=\frac{k \sqrt{2 a}}{\sqrt{-\gamma}} \int \frac{d u}{u^{2}+\left(a_{1} / a_{2}\right) u} .
$$

Integrating (21) by using Mathematica programming, we obtain the solutions to (1), as follows, for different values of the roots of the polynomial equation:

$$
\begin{gathered}
\pm\left(\mu-\mu_{0}\right)=-\frac{A}{u-\alpha_{1}}, \quad \alpha_{1}=\alpha_{2}, \\
\pm\left(\mu-\mu_{0}\right)=\frac{A}{\alpha_{1}-\alpha_{2}} \ln \left|\frac{u-\alpha_{1}}{u-\alpha_{2}}\right|, \quad \alpha_{1} \neq \alpha_{2},
\end{gathered}
$$

where $A= \pm k \sqrt{2 a} / \sqrt{-\gamma}$, and also $\alpha_{1}$ and $\alpha_{2}$ are the roots of the polynomial equation as follows:

$$
u^{2}+\frac{a_{1}}{a_{2}} u=0 .
$$

Therefore, we find solutions

$$
u(x, t)=\alpha_{1}+\frac{A}{ \pm\left(k x-\left(3 k \sqrt{\beta a} t^{\alpha} / \sqrt{2} \Gamma(1+\alpha)\right)-\eta_{0}\right)},
$$

$$
\begin{aligned}
& u(x, t) \\
& =\alpha_{1} \pm \frac{\alpha_{1}-\alpha_{2}}{\exp \left[\left(\left(\alpha_{1}-\alpha_{2}\right) / A\right)\left(k x-\left(3 k \sqrt{\beta a} t^{\alpha} / \sqrt{2} \Gamma(1+\alpha)\right)-\eta_{0}\right)\right]-1} .
\end{aligned}
$$

For simplicity, if we take $\eta_{0}=0$, then the solutions equations (24) and (25) can reduce to rational and single kink solution, respectively,

$$
\begin{gathered}
u(x, t)=\alpha_{1}+\frac{A}{B_{1}\left(x-\lambda_{1} t^{\alpha}\right)}, \\
u(x, t)=\alpha_{1} \pm \frac{\alpha_{1}-\alpha_{2}}{\exp \left[B_{2}\left(x-\lambda_{1} t^{\alpha}\right)\right]-1},
\end{gathered}
$$

where $B_{1}= \pm k, B_{2}=k\left(\alpha_{1}-\alpha_{2}\right) / A$, and $\lambda_{1}=3 \sqrt{\beta a} / \sqrt{2} \Gamma(1+$ $\alpha)$. Here, $B_{1}$ and $B_{2}$ are the inverse width of the soliton. We can regulate (27) to rewrite in the hyperbolic form as follows:

$$
\begin{aligned}
& u(x, t)=\alpha_{1}+\frac{\alpha_{1}-\alpha_{2}}{\exp \left[B_{2}\left(x-\lambda_{1} t^{\alpha}\right)\right]-1}, \\
& u(x, t)=\alpha_{2}+\frac{\alpha_{2}-\alpha_{1}}{\exp \left[B_{2}\left(x-\lambda_{1} t^{\alpha}\right)\right]-1} .
\end{aligned}
$$

If we consider the following equation for simplicity of (28):

$$
u(\mu)=\alpha_{1}+\frac{\alpha_{1}-\alpha_{2}}{\exp [\mu]-1},
$$

then, we get

$$
\begin{aligned}
u(\mu) & =\alpha_{1}+\frac{\alpha_{1}-\alpha_{2}}{\exp [\mu]-1}=\frac{\alpha_{1} \exp [\mu]-\alpha_{2}}{\exp [\mu]-1} \\
& =\alpha_{1} \frac{\exp [\mu]-\alpha_{2} / \alpha_{1}}{\exp [\mu]-1} .
\end{aligned}
$$


If it takes $\alpha_{1}=-\alpha_{2}$ for (28), we get the hyperbolic function solution of (28):

$$
u(\mu)=\alpha_{1} \operatorname{coth}\left[\frac{\mu}{2}\right]
$$

where $\mu=B_{2}\left(x-\lambda_{1} t^{\alpha}\right)$.

Remark 2. The solutions equations (26)-(27) obtained by using the extended trial equation method for (1) have been checked by Mathematica. To our knowledge, the rational function solution and single kink solution that we found in this paper are not shown in the previous literature. These results are new traveling wave solutions of (1).

Case 2. In the same way as in Case 1, If we take $l=1$ and $n=3$, then

$$
\begin{gathered}
u^{\prime}=\frac{a_{0}+a_{1} u+a_{2} u^{2}+a_{3} u^{3}}{b_{0}+b_{1} u}, \\
u^{\prime \prime}=\left(\left(a_{0}+a_{1} u+a_{2} u^{2}+a_{3} u^{3}\right)\right. \\
\times\left(\left(b_{0}+b_{1} u\right)\left(a_{1}+2 a_{2} u+3 a_{3} u^{2}\right)\right. \\
\left.\left.\quad-b_{1}\left(a_{0}+a_{1} u+a_{2} u^{2}+a_{3} u^{3}\right)\right)\right) \\
\times\left(\left(b_{0}+b_{1} u\right)^{3}\right)^{-1}
\end{gathered}
$$

where $a_{3} \neq 0, b_{1} \neq 0$. Respectively, solving the algebraic equation system (11) yields the following:

$$
\begin{aligned}
& a_{0}=0, \quad a_{1}= \pm \frac{\sqrt{\beta} b_{0}}{k \sqrt{2 a}}, \\
& a_{2}=-\frac{b_{0} \sqrt{(-\gamma)}-b_{1} \sqrt{\beta}}{k \sqrt{2 a}}, \\
& a_{3}=-\frac{b_{1} \sqrt{\gamma}}{k \sqrt{2 a}}, \quad b_{0}=b_{0}, \\
& b_{1}=b_{1}, \quad \lambda= \pm \frac{3 k \sqrt{\beta a}}{\sqrt{2}} .
\end{aligned}
$$

Substituting these coefficients into (12), we have

$$
\pm\left(\mu-\mu_{0}\right)=-\frac{\sqrt{\gamma}}{k \sqrt{2 a}} \int \frac{u+b_{0} / b_{1}}{u^{3}+\left(a_{2} / a_{3}\right) u^{2}+\left(a_{1} / a_{3}\right) u} d u .
$$

Integrating (35), we procure the solution to (1) as follows:

$$
\pm\left(\mu-\mu_{0}\right)=-\frac{A_{1}\left(b_{0}+2 b_{1} u-b_{1} \alpha_{1}\right)}{2 b_{1}\left(u-\alpha_{1}\right)^{2}}
$$

$$
\begin{aligned}
\pm\left(\mu-\mu_{0}\right)= & \frac{A_{1}}{b_{1}}\left(\frac{b_{0}+b_{1} \alpha_{2}}{\left(\alpha_{1}-\alpha_{2}\right)^{2}} \ln \left|\frac{u-\alpha_{2}}{u-\alpha_{1}}\right|\right. \\
& \left.-\frac{b_{0}+b_{1} \alpha_{1}}{\left(u-\alpha_{1}\right)\left(\alpha_{1}-\alpha_{2}\right)}\right), \\
\pm\left(\mu-\mu_{0}\right)= & \frac{A_{1}}{b_{1}\left(\alpha_{1}-\alpha_{2}\right)\left(\alpha_{2}-\alpha_{3}\right)\left(\alpha_{1}-\alpha_{3}\right)} \\
& \times\left(\ln \left|\frac{\left(u-\alpha_{1}\right)^{M}\left(u-\alpha_{3}\right)^{N}}{\left(u-\alpha_{2}\right)^{P}}\right|\right),
\end{aligned}
$$

where $A_{1}=-\sqrt{\gamma} / k \sqrt{2 a}, M=\left(\alpha_{2}-\alpha_{3}\right)\left(b_{0}+b_{1} \alpha_{1}\right), N=$ $\left(\alpha_{1}-\alpha_{2}\right)\left(b_{0}+b_{1} \alpha_{3}\right)$ and $P=\left(\alpha_{1}-\alpha_{3}\right)\left(b_{0}+b_{1} \alpha_{2}\right)$. Also $\alpha_{1}, \alpha_{2}$, and $\alpha_{3}$ are the roots of the polynomial equation

$$
u^{3}+\frac{a_{2}}{a_{3}} u^{2}+\frac{a_{1}}{a_{3}} u+\frac{a_{0}}{a_{3}}=0 .
$$

Therefore, we find a solution from (36):

$$
\begin{aligned}
& u(x, t) \\
& =\left(b_{1}\left(2 \alpha_{1}\left(k x \pm \frac{3 k t^{\alpha} \sqrt{\beta a}}{\sqrt{2} \Gamma(1+\alpha)}-\eta_{0}\right)-A_{1}\right)\right. \\
& \left.\quad+\sqrt{A_{1} b_{1}\left(A_{1} b_{1}-2\left(b_{0}+b_{1} \alpha_{1}\right)\left(k x \pm \frac{3 k t^{\alpha} \sqrt{\beta a}}{\sqrt{2} \Gamma(1+\alpha)}-\eta_{0}\right)\right)}\right) \\
& \quad \times\left(2 b_{1}\left(k x \pm \frac{3 k t^{\alpha} \sqrt{\beta a}}{\sqrt{2} \Gamma(1+\alpha)}-\eta_{0}\right)\right)^{-1} .
\end{aligned}
$$

For simplicity, if we take $\eta_{0}=0$, then the solution equation (39) can reduce to rational solution

$$
\begin{aligned}
u(x, t)= & \left(b_{1}\left(2 \alpha_{1}\left(k x \pm k \lambda_{1} t^{\alpha}\right)-A_{1}\right)\right. \\
& \left.\quad+\sqrt{A_{1} b_{1}\left(A_{1} b_{1}-2\left(b_{0}+b_{1} \alpha_{1}\right)\left(k x \pm k \lambda_{1} t^{\alpha}\right)\right)}\right) \\
& \times\left(2 b_{1}\left(k x \pm k \lambda_{1} t^{\alpha}\right)\right)^{-1} .
\end{aligned}
$$

Remark 3. The solution equation (40) computed in Case 2 has been checked by Mathematica. We think that these solutions have not been found in the literature, and these results are new traveling wave solutions of (1).

Example 4. Secondly, we consider the time fractional generalized Burgers equation [24] as follows:

$$
\frac{\partial^{\alpha} u}{\partial t^{\alpha}}-u_{x x}-\beta u^{p} u_{x}=0 .
$$

In the case of $\alpha=1$ and $p=1$, (41) reduces to the well-known classical nonlinear Burgers equation. Many researchers have tried to get the exact solutions of this equation by using a different method [28, 29].

Let us consider the travelling wave solutions of (41), and then we perform the transformation $u(x, t)=u(\eta)$ and 
$\eta=k x-\lambda t^{\alpha} / \Gamma(1+\alpha)$ where $k, \lambda$ are constants. Then, integrating this equation with respect to $\eta$ and setting the integration constant to zero, we get

$$
-\lambda u^{\prime}(\eta)-k^{2} u^{\prime \prime}(\eta)-\beta k u^{p}(\eta) u_{x}(\eta)=0 .
$$

When we conduct once more transformation

$$
u(\eta)=v^{1 / p}(\eta)
$$

we get the following:

$$
\lambda p(p+1) v-k^{2}(p+1) v^{\prime}-\beta k v^{2}=0 .
$$

Substituting (8) into (44) and using balance principle yield the following:

$$
n=l+2 \text {. }
$$

This resolution procedure is applied, and we obtain results as follows.

Case 1. If we take $l=0$ and $n=2$, then

$$
v^{\prime}=\frac{a_{0}+a_{1} v+a_{2} v^{2}}{b_{0}},
$$

where $a_{2} \neq 0$ and $b_{0} \neq 0$. Thus, we have a system of algebraic equations from the coefficients of the polynomial of $v$. Solving the algebraic equation system (11) yields the following:

$$
\begin{gathered}
a_{0}=0, \quad a_{1}=a_{1}, \quad a_{2}=-\frac{p \beta b_{0}}{k+k p}, \\
b_{0}=b_{0}, \quad \lambda=-\frac{k^{2} a_{1}}{p b_{0}} .
\end{gathered}
$$

By substituting these coefficients into (11), we have

$$
\pm\left(\mu-\mu_{0}\right)=-\frac{k+k p}{p \beta} \int \frac{d v}{v^{2}+\left(a_{1} / a_{2}\right) v} .
$$

By integrating (48), we procure the solution to (41) as follows:

$$
\begin{gathered}
\pm\left(\mu-\mu_{0}\right)=-\frac{A_{2}}{v-\alpha_{1}}, \\
\pm\left(\mu-\mu_{0}\right)=\frac{A_{2}}{\alpha_{1}-\alpha_{2}} \ln \left|\frac{v-\alpha_{1}}{v-\alpha_{2}}\right|,
\end{gathered}
$$

where $A_{2}=-(k+k p) / p \beta$. By substituting the solutions equation (49) into (43), we found solutions of the following exact traveling wave solutions, such as rational function solution and single kink solution:

$$
\begin{gathered}
u(x, t)=\left[\alpha_{1} \pm \frac{A_{2}}{\left(k x+\left(k a_{1} t^{\alpha} / p b_{0} \Gamma(1+\alpha)\right)-\eta_{0}\right)}\right]^{1 / p}, \\
u(x, t) \\
=\left[\alpha_{1} \pm \frac{\alpha_{1}-\alpha_{2}}{\exp \left[\left(\left(\alpha_{1}-\alpha_{2}\right) / A_{2}\right)\left(k x+\left(k^{2} a_{1} t^{\alpha} / p b_{0} \Gamma(1+\alpha)\right)-\eta_{0}\right)\right]-1}\right]^{1 / p} .
\end{gathered}
$$

For simplicity, if we take $\eta_{0}=0$, then the solutions equation (50) can reduce to the following:

$$
u(x, t)=\left[\alpha_{1} \pm \frac{B_{3}}{\left(x-\lambda_{2} t^{\alpha}\right)}\right]^{1 / p}
$$

$$
u(x, t)=\left[\alpha_{1} \pm \frac{\alpha_{1}-\alpha_{2}}{\exp \left[B_{4}\left(x-\lambda_{2} t^{\alpha}\right)\right]-1}\right]^{1 / p}
$$

where $B_{3}=A_{2} / k, B_{4}=k\left(\alpha_{1}-\alpha_{2}\right) / A_{2}$, and $\lambda_{2}=-k a_{1} / p b_{0} \Gamma$ $(1+\alpha)$.

Remark 5. The solutions equations (51) and (52) obtained by using the modified trial equation method for (41) have been checked by Mathematica. To our knowledge, the rational function solution and single kink solution that we found in this paper are new traveling wave solutions of (41).

Case 1. If we take $l=1$ and $n=3$, then

$$
v^{\prime}=\frac{a_{0}+a_{1} v+a_{2} v^{2}+a_{3} v^{3}}{b_{0}+b_{1} v}
$$

where $a_{3} \neq 0, b_{1} \neq 0$. Thus, we have a system of algebraic equations from the coefficients of the polynomial of $v$. Solving the algebraic equation system (11) yields the following:

$$
\begin{gathered}
a_{0}=0, \quad a_{1}=a_{1}, \quad a_{2}=a_{2}, \\
a_{3}=-\frac{p \beta b_{0}\left(k(1+p) a_{2}+p \beta b_{0}\right)}{k^{2}(1+p)^{2} a_{1}}, \\
b_{0}=b_{0}, \\
b_{1}=-\frac{b_{0}\left(k(1+p) a_{2}+p \beta b_{0}\right)}{k(1+p) a_{1}}, \\
\lambda=-\frac{k^{2} a_{1}}{p b_{0}} .
\end{gathered}
$$

By substituting these coefficients into (11), we have

$$
\pm\left(\mu-\mu_{0}\right)=\frac{k(1+p)}{p \beta} \int \frac{v+b_{0} / b_{1}}{v^{3}+\left(a_{2} / a_{3}\right) v^{2}+\left(a_{1} / a_{3}\right) v} d v
$$

where $A_{3}=(k+k p) / p \beta$. By integrating (55), we procure the solution to (41) as follows:

$$
\pm\left(\mu-\mu_{0}\right)=-\frac{A_{3}\left(b_{0}+2 b_{1} v-b_{1} \alpha_{1}\right)}{2 b_{1}\left(v-\alpha_{1}\right)^{2}},
$$




$$
\begin{aligned}
\pm\left(\mu-\mu_{0}\right)= & \frac{A_{3}}{b_{1}}\left(\frac{b_{0}+b_{1} \alpha_{2}}{\left(\alpha_{1}-\alpha_{2}\right)^{2}} \ln \left|\frac{v-\alpha_{2}}{v-\alpha_{1}}\right|\right. \\
& \left.-\frac{b_{0}+b_{1} \alpha_{1}}{\left(v-\alpha_{1}\right)\left(\alpha_{1}-\alpha_{2}\right)}\right), \\
\pm\left(\mu-\mu_{0}\right)= & \frac{A_{3}}{b_{1}\left(\alpha_{1}-\alpha_{2}\right)\left(\alpha_{2}-\alpha_{3}\right)\left(\alpha_{1}-\alpha_{3}\right)} \\
& \times\left(\ln \left|\frac{\left(v-\alpha_{1}\right)^{M}\left(v-\alpha_{3}\right)^{N}}{\left(v-\alpha_{2}\right)^{P}}\right|\right),
\end{aligned}
$$

where $A_{3}=k(1+p) / p \beta, M=\left(\alpha_{2}-\alpha_{3}\right)\left(b_{0}+b_{1} \alpha_{1}\right), N=$ $\left(\alpha_{1}-\alpha_{2}\right)\left(b_{0}+b_{1} \alpha_{3}\right)$, and $P=\left(\alpha_{1}-\alpha_{3}\right)\left(b_{0}+b_{1} \alpha_{2}\right)$. Also $\alpha_{1}, \alpha_{2}$, and $\alpha_{3}$ are the roots of the polynomial equation:

$$
v^{3}+\frac{a_{2}}{a_{3}} v^{2}+\frac{a_{1}}{a_{3}} v+\frac{a_{0}}{a_{3}}=0 .
$$

By substituting the solution equation (56) into (43), we found solution of the following exact traveling wave solutions, such as rational function solution:

$$
\begin{aligned}
& u(x, t) \\
& =\left[\left(b_{1}\left(2 \alpha_{1}\left(k x+\frac{k^{2} a_{1} t^{\alpha}}{p b_{0} \Gamma(1+\alpha)}-\eta_{0}\right)-A_{3}\right)\right.\right. \\
& \left.\quad+\sqrt{A_{3} b_{1}\left(A_{3} b_{1}-2\left(b_{0}+b_{1} \alpha_{1}\right)\left(k x+\frac{k^{2} a_{1} t^{\alpha}}{p b_{0} \Gamma(1+\alpha)}-\eta_{0}\right)\right)}\right) \\
& \left.\quad \times\left(2 b_{1}\left(k x+\frac{k^{2} a_{1} t^{\alpha}}{p b_{0} \Gamma(1+\alpha)}-\eta_{0}\right)\right)^{-1}\right]^{1 / p} .
\end{aligned}
$$

For simplicity, if we take $\eta_{0}=0$, then the solution equation (59) can reduce to rational solution:

$$
\begin{aligned}
u(x, t)=[ & \left(b_{1}\left(2 \alpha_{1}\left(k x \pm k \lambda_{2} t^{\alpha}\right)-A_{3}\right)\right. \\
& \left.+\sqrt{A_{3} b_{1}\left(A_{3} b_{1}-2\left(b_{0}+b_{1} \alpha_{1}\right)\left(k x \pm k \lambda_{2} t^{\alpha}\right)\right)}\right) \\
& \left.\times\left(2 b_{1}\left(k x \pm k \lambda_{2} t^{\alpha}\right)\right)^{-1}\right]^{1 / p} .
\end{aligned}
$$

Remark 6. The solution equation (60) computed in Case 2 has been checked by Mathematica. We think that these solutions have not been found in the literature, and these results are new traveling wave solutions of (41).

\section{Conclusions}

In this paper, the modified trial equation method has been applied to the one-dimensional nonlinear fractional wave equation and time fractional generalized Burgers equation. We used it to obtain some soliton and rational function solutions to the one-dimensional nonlinear fractional wave equation and time fractional generalized Burgers equation.
This method is reliable and effective and gives several new solution functions such as rational function solutions and single kink solutions. We think that the proposed method can also be applied to other generalized fractional nonlinear differential equations. In our future studies, we will solve nonlinear fractional partial differential equations by this approach.

\section{References}

[1] K. S. Miller and B. Ross, An Introduction to the Fractional Calculus and Fractional Differential Equations, John Wiley \& Sons, New York, NY, USA, 1993.

[2] A. A. Kilbas, H. M. Srivastava, and J. J. Trujillo, Theory and Applications of Fractional Differential Equations, vol. 204 of North-Holland Mathematics Studies, Elsevier Science B.V., Amsterdam, The Netherlands, 2006.

[3] I. Podlubny, Fractional Differential Equations, vol. 198 of Mathematics in Science and Engineering, Academic Press, San Diego, Calif, USA, 1999.

[4] H. Bulut, H. M. Baskonus, and S. Tuluce, "The solution of wave equations by Sumudu transform method," Journal of Advanced Research in Applied Mathematics, vol. 4, no. 3, pp. 66-72, 2012.

[5] H. Bulut, H. M. Baskonus, and S. Tuluce, "Homotopy perturbation Sumudu transform for heat equations," Mathematics in Engineering, Science and Aerospace, vol. 4, no. 1, pp. 49-60, 2013.

[6] M. Safari, D. D. Ganji, and M. Moslemi, "Application of He's variational iteration method and Adomian's decomposition method to the fractional KdV-Burgers-Kuramoto equation," Computers \& Mathematics with Applications, vol. 58, no. 11-12, pp. 2091-2097, 2009.

[7] L. Song and H. Zhang, "Solving the fractional BBM-Burgers equation using the homotopy analysis method," Chaos, Solitons \& Fractals, vol. 40, no. 4, pp. 1616-1622, 2009.

[8] S. Abbasbandy and A. Shirzadi, "Homotopy analysis method for multiple solutions of the fractional Sturm-Liouville problems," Numerical Algorithms, vol. 54, no. 4, pp. 521-532, 2010.

[9] K. A. Gepreel, "The homotopy perturbation method applied to the nonlinear fractional Kolmogorov-Petrovskii-Piskunov equations," Applied Mathematics Letters, vol. 24, no. 8, pp. 14281434, 2011.

[10] H. Jafari and S. Momani, "Solving fractional diffusion and wave equations by modified homotopy perturbation method," Physics Letters A, vol. 370, no. 5-6, pp. 388-396, 2007.

[11] W. Mitkowski, "Approximation of fractional diffusion-wave equation," Acta Mechanica et Automatica, vol. 5, no. 2, pp. 6568, 2011.

[12] X. Zhang, J. Liu, J. Wen, B. Tang, and Y. He, "Analysis for onedimensional time-fractional Tricomi-type equations by LDG methods," Numerical Algorithms, vol. 63, no. 1, pp. 143-164, 2013.

[13] M. G. Sakar, F. Erdogan, and A. Yldrm, "Variational iteration method for the time-fractional Fornberg-Whitham equation," Computers and Mathematics with Applications, vol. 63, no. 9, pp. 1382-1388, 2012.

[14] C.-S. Liu, "A new trial equation method and its applications," Communications in Theoretical Physics, vol. 45, no. 3, pp. 395397, 2006.

[15] C.-S. Liu, "Trial equation method to nonlinear evolution equations with rank inhomogeneous: mathematical discussions and its applications," Communications in Theoretical Physics, vol. 45, no. 2, pp. 219-223, 2006. 
[16] Y.-J. Cheng, "Classification of traveling wave solutions to the Vakhnenko equations," Computers \& Mathematics with Applications, vol. 62, no. 10, pp. 3987-3996, 2011.

[17] Y. Gurefe, A. Sonmezoglu, and E. Misirli, "Application of the trial equation method for solving some nonlinear evolution equations arising in mathematical physics," Pramana-Journal of Physics, vol. 77, no. 6, pp. 1023-1029, 2011.

[18] H. Bulut and B. Kilic, "Exact solutions for some fractional nonlinear partial differential equations via Kudryashov method," $E$ Journal of New World Sciences Academy, vol. 8, no. 1, pp. 24-31, 2013.

[19] H. Bulut and B. Kilic, "Finding exact solution by using a new auxiliary equation for fractional RLW burges equation," $E$ Journal of New World Sciences Academy, vol. 8, no. 1, pp. 1-10, 2013.

[20] Y. Pandir, Y. Gurefe, U. Kadak, and E. Misirli, "Classification of exact solutions for some nonlinear partial differential equations with generalized evolution," Abstract and Applied Analysis, vol. 2012, Article ID 478531, 16 pages, 2012.

[21] Y. Gurefe, E. Misirli, A. Sonmezoglu, and M. Ekici, "Extended trial equation method to generalized nonlinear partial differential equations," Applied Mathematics and Computation, vol. 219, no. 10, pp. 5253-5260, 2013.

[22] Y. Pandir, Y. Gurefe, and E. Misirli, "Classification of exact solutions to the generalized Kadomtsev-Petviashvili equation," Physica Scripta, vol. 87, pp. 1-12, 2013.

[23] H. Jafari and S. Seifi, "Homotopy analysis method for solving linear and nonlinear fractional diffusion-wave equation," Communications in Nonlinear Science and Numerical Simulation, vol. 14, no. 5, pp. 2006-2012, 2009.

[24] R. Sahadevan and T. Bakkyaraj, "Invariant analysis of time fractional generalized Burgers and Korteweg-de Vries equations," Journal of Mathematical Analysis and Applications, vol. 393, no. 2, pp. 341-347, 2012.

[25] G. Jumarie, "Modified Riemann-Liouville derivative and fractional Taylor series of nondifferentiable functions further results," Computers \& Mathematics with Applications, vol. 51, no. 9-10, pp. 1367-1376, 2006.

[26] G. Jumarie, "Fractional Hamilton-Jacobi equation for the optimal control of nonrandom fractional dynamics with fractional cost function," Journal of Applied Mathematics \& Computing, vol. 23, no. 1-2, pp. 215-228, 2007.

[27] G. Jumarie, "Table of some basic fractional calculus formulae derived from a modified Riemann-Liouville derivative for nondifferentiable functions," Applied Mathematics Letters, vol. 22, no. 3, pp. 378-385, 2009.

[28] P. L. Sachdev and Ch. Srinivasa Rao, "N-wave solution of modified Burgers equation," Applied Mathematics Letters, vol. 13, no. 4, pp. 1-6, 2000.

[29] A. Biswas, H. Triki, T. Hayat, and O. M. Aldossary, "1-soliton solution of the generalized Burgers equation with generalized evolution," Applied Mathematics and Computation, vol. 217, no. 24, pp. 10289-10294, 2011. 


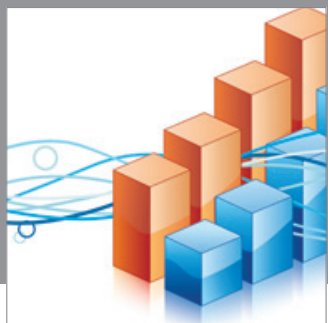

Advances in

Operations Research

mansans

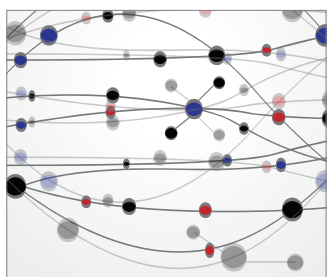

The Scientific World Journal
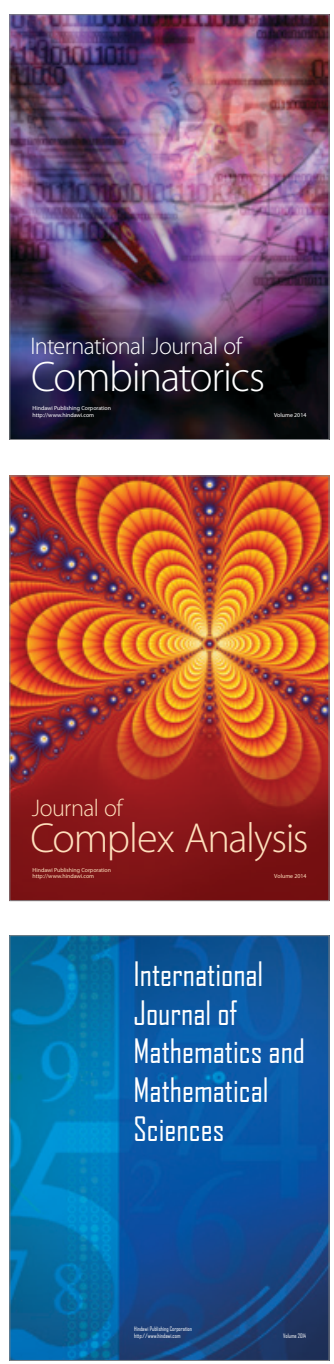
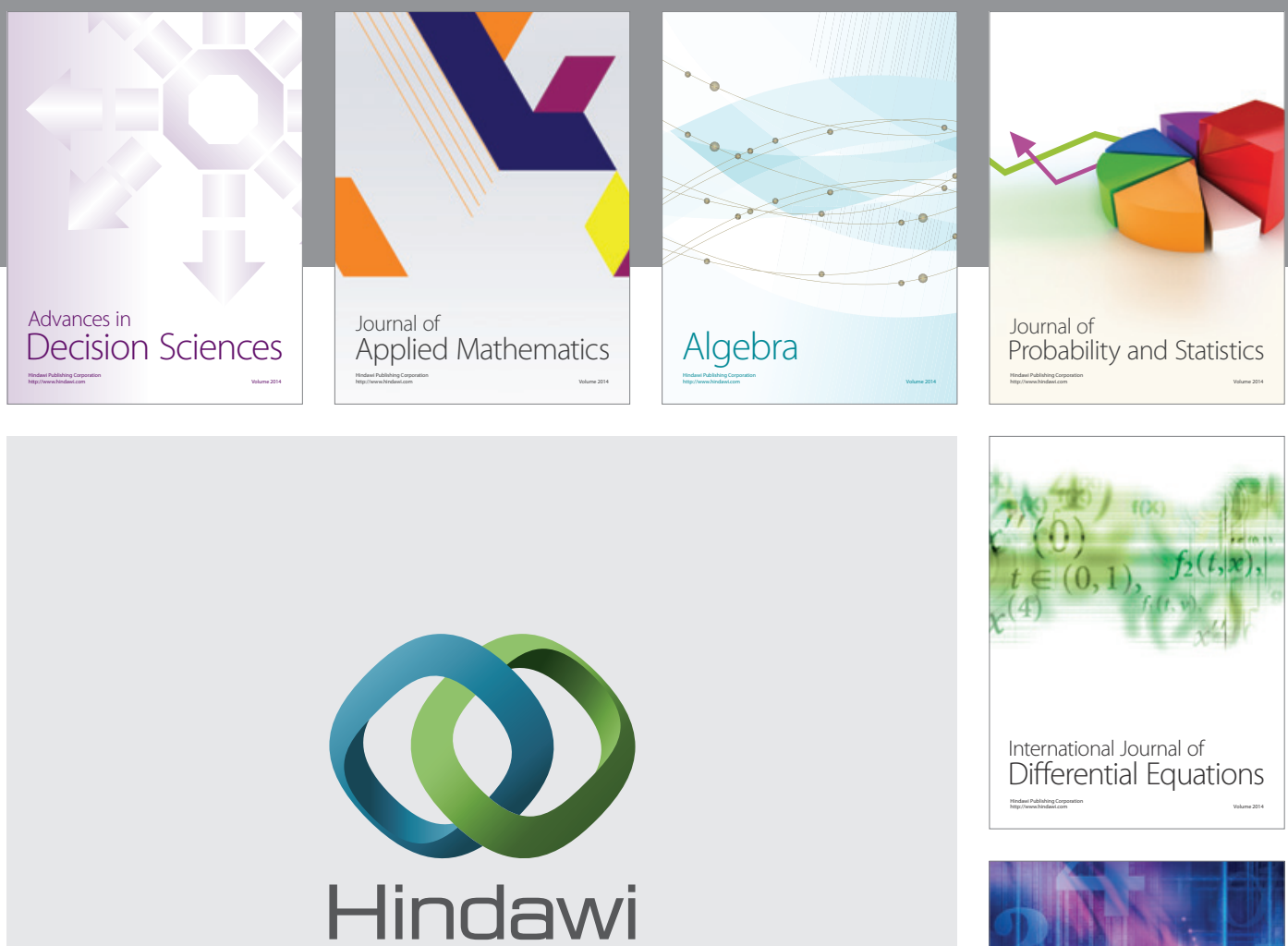

Submit your manuscripts at http://www.hindawi.com
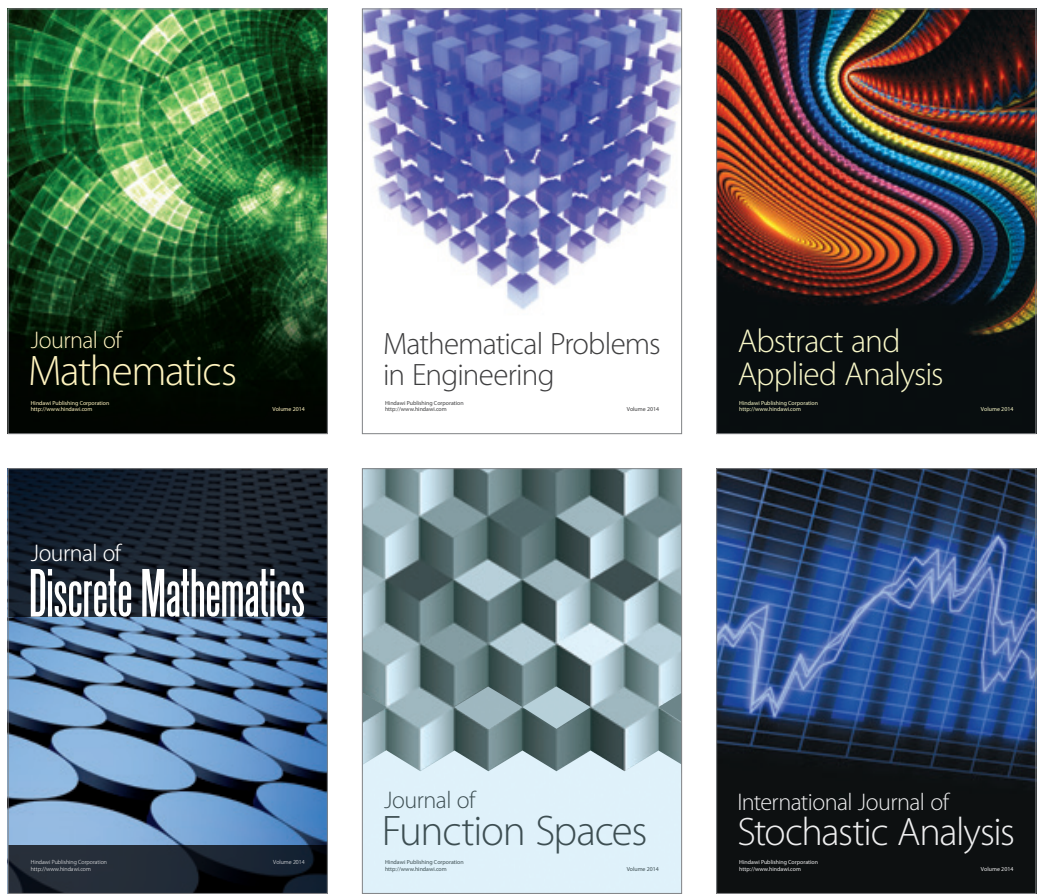

Journal of

Function Spaces

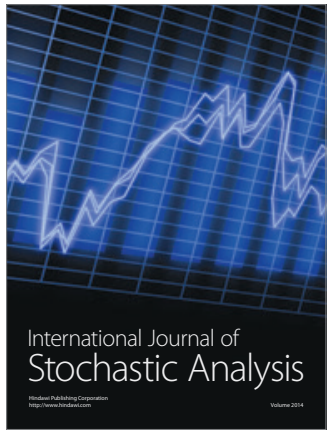

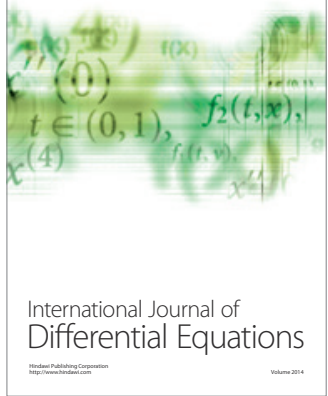
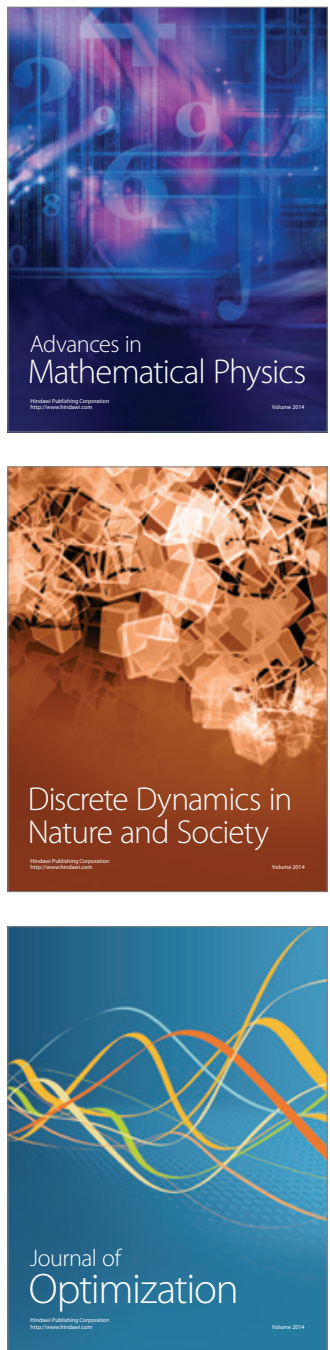